\title{
NATURAL ANTIOXIDANT PRODUCTION BY Oscillatoria sp. AND Microcystis sp. FROM PONDS OF PURI DISTRICT, ODISHA
}

\author{
PRADYUTI DASH ${ }^{*}$ AND PADHI S.B. ${ }^{2}$
}

\author{
${ }^{1}$ Algal Research Laboratory, Department of Botany, Berhampur University, Berhampur-760 007, Orissa, India. \\ ${ }^{2}$ CES, Govt. of Odisha, Department of Botany, Berhampur University, Berhampur-760 007, Orissa, India. \\ ${ }^{*}$ Corresponding Author: Email- pradyutidash@gmail.com
}

Received: June 20, 2012; Accepted: July 03, 2012

\begin{abstract}
The implications of diet on health sustainability have assumed a major importance. Microcystis are able to enhance the nutritional content of conventional food and feed preparation and hence to positively affect humans and animal health due to their original chemical compositions, protein contents, antioxidant properties, vitamins and other biologically active compounds. Butylated hydroxytoluene 'BHT' is one of the synthetic antioxidant agents commonly used for food additives. Cyanobactria like Oscillatoria sp. and Microcystis aerungionosa, those are commonly available in the coastal zone of Puri District of Odisha State were capable of producing this compound. Extracts from these two species exhibited various degrees of antioxidant properties when they were tested with free radical scavenging assay. The highest antioxidant activity was observed in the extracts of Microcystic aerungionosa, which displayed a similar activity to synthetic BHT. Gas chromatography and mass spectroscopy analysis concluded that the compounds are very similar to the synthetic BHT. This synthetic antioxidant was produced in the cells irradiated with higher light intensity. More BHT was produced in the cells irradiated with a higher light intensity and its production was irradiance dependent. The quantity of cellular BHT displayed a positive correlation with antioxidant activity of the tested species. The present study confirms the production of natural antioxidant like BHT from these two species of Puri District of Odisha State which constitute a potential source for producing natural antioxidant.
\end{abstract}

Keywords- Butylated hydroxytoluene, cyanobacteria, green alga, irradiance, natural antioxidant.

Citation: Pradyuti Dash and Padhi S.B. (2012) Natural Antioxidant Production by Oscillatoria Sp. and Microcystis Sp. From Ponds of Puri District, Odisha. International Journal of Microbiology Research, ISSN: 0975-5276 \& E-ISSN:0975-9174, Volume 4, Issue 6, pp.-249-252.

Copyright: Copyright@2012 Pradyuti Dash and Padhi S.B. This is an open-access article distributed under the terms of the Creative Commons Attribution License, which permits unrestricted use, distribution and reproduction in any medium, provided the original author and source are credited.

\section{Introduction}

Lipid peroxidation is mostly an undesirable deteriorative reaction in food materials, which can produce rancid odors and flavors during storage and processing. Moreover the nutritional quality and safety of foods also can easily change due to their toxic compound formation $[1,2]$.These lipid peroxidation by-products, called free radicals, give damage to healthy cells and thereby create harmful molecules. Free radicals and other reactive oxygen species (ROS) are generated continuously not only via normal physiological processes but also by external stimulations. Normal physiological processes need oxygen in order to carry out their operations as a resultant by-product like ROS are produced within the human body. If these harmful factors accumulate in cell, tissue and other vital organs of the body, then our body exposed to dangerous circumstances of the external stimulation, ROS can be induced by tobacco smoke, ionizing radiation, certain pollutants, organic solvents and pesticides [3,4]. ROS including superoxide $\left(\mathrm{O}_{2} \cdot-\right)$, hydroxyl radical $(\mathrm{HO} \cdot)$ and hydrogen peroxide $\left(\mathrm{H}_{2} \mathrm{O}_{2}\right)$, have the ability to react with a large variety of easily oxidizable cellular components, such as proteins, lipids, nucleic acids and carbohydrates. Their oxidative damages cause aging and many other diseases, including arthritis, strokes, heart diseases, atherosclerosis, diabetes, cancers and neurodegenerative disorders $[5,6]$. Reactive oxygen species (ROS), such as the superoxide anion, 
hydroxyl radicals and hydrogen peroxide, cause lipid oxidation and thereby decrease the nutritional value and appearance of food. In addition, ROS are the major cause of qualitative decay of food, which leads to rancidity, toxicity and destruction of major biochemical components that are important in metabolism [7].

Antioxidants are important inhibitors against lipid peroxidation, therefore these compounds are utilized to delay or prevent lipid peroxidation in foods and the oxidation of cellular substrates. They exert their effects by scavenging ROS, activating a battery of detoxifying proteins, or by preventing the generation of ROS [8]. The most commonly used antioxidants are butylated hydroxyanisole $(\mathrm{BHA})$, butylated hydroxytoluene $(\mathrm{BHT})$, tert-butylhydroquinone (TBHQ) and propyl gallate (PG). However, there has been growing concern over their safety and toxicity. Moreover, some studies [9] have been reported that there is an inverse relationship between dietary intake of antioxidant-rich foods and the incidence of human diseases. Therefore, development and utilization of more effective antioxidant from natural resources are desired for use in foods or medicinal materials to replace the synthetic antioxidants. Modern food industry leads to an increase of cheaper, healthier and more convenient products. The use of natural ingredients, like polyunsaturated fatty acids (PUFA's) and antioxidant pigments, exhibiting high impact on functional properties are important to reduce chronic diseases incidence, which are strongly considered of capital importance in Europe, where aging population and welfare costs are fatal for public resources management. The impact of natural substances introduced in the diet via "usual" foods is proved to be efficient at long term and do not present the drawbacks of traditional therapeutic actions based on medicines of short term impact.

Microalgae are able to enhance the nutritional content of conventional food and feed preparation and hence to positively affect humans and animal health due to their original chemical composition, namely high protein content, with balanced amino acids pattern, carotenoids, fatty acids, vitamins, polysaccharides, sterols, phycobilins and other biologically active compounds, more efficiently than traditional crops. Algae are photosynthetic organisms, which possess reproductive simple structures. These organisms constitute a total of $25-30,000$ species, with a great diversity of forms and sizes and that can exist from unicellular microscopic organisms (microalgae) to multicellular of great size (macroalgae). Algae can be a very interesting natural source of new compounds with biological activity that could be used as functional ingredients. Some important algae aspects, such as natural character, easy cultivation, their rapid growing (for many of the species) and the possibility of controlling the production of some bioactive compounds by manipulating the cultivation conditions. In this way, algae can be considered as genuine natural reactors

Algae are receiving increasing attention as possible antioxidant source for human civilization. Some of the most biotechnologically relevant microalgae are the green algae like Oscillatoria sp.and Microcystis sp.can be be used as nutritional supplements for humans and animal additives. Microalgae can be used to enhance the nutritional value of food and animal feed due to their well balanced chemical composition, antioxidant properties. Oscillatoria sp. and Microcystis sp. from Puri District, Odisha may therefore become an interestingly source enormous biological resources, representing one of the most promising sourcesfor new products and applications for the production of natural antioxidants like BHT (butylated hydroxyltoluene).

\section{Materials and Method Chemicals Required}

Ferrozine, linoleic acid, trichloroacetic acid (TCA), DPPH, potassium ferricyanide and hydrogen peroxide. Gallic acid, quercetin, butylated hydroxyanisole (BHA), ascorbic acid, sulfanilamide, $\mathrm{N}$-(1 -naphthyl) ethylenediamine dihydrochloride, EDTA, ferric chloride, Muller Hinton Agar, Nutrient broth.

\section{Collection and Preparation of Sample}

Microcystis $s p$. and oscillatoria $s p$. were collected manually from the ponds of Puri district. The harvested macroalgae were stored in plastic bags for transportation to the laboratory.

\section{Collection and Preparation of Algal Extracts}

Dried materials were coarsely ground before extraction. $5 \mathrm{~g}$ of dried materials were extracted by maceration with $70 \%$ ethanol (1 h sonication, filtered; repeated 2 times). The extract was then separated from the sample residue by filtration through Whatman No.1 filter paper. The resultant extracts were concentrated in a rotary evaporator under reduced pressure until a crude solid extract was obtained, which were then freeze-dried for complete solvent which were then freeze-dried for complete solvent removal (0.9 g).

\section{Partial Purification of Extracts by TLC}

Extracts of samples were used for partial purification through silica gel TLC. The dried crude extracts were dissolved in a small volume of chloroform, methanol $(1,1 \mathrm{v} / \mathrm{v})$, applied to the TLC plates and chromatographed using acetone, methanol, water (40:20:1 v/ $\mathrm{v} / \mathrm{v}$ ) as the developing solvents. Bands with different Rf values were scraped out separately and extracted according to Bligh and Dyer (1959) [10] and centrifuged. The desired ratio of solvents was obtained by adding the appropriate volume of chloroform and water to arrive at a final ratio of chloroform, methanol, water $(1: 1: 0.9 \mathrm{v} / \mathrm{v} / \mathrm{v})$. The chloroform extracts were evaporated to dryness. The partially purified dried fractions of all of all samples were tested for antioxidant activities.

\section{Determination of Total Phenolic Compounds and Flavonoid Content}

Total phenolic compound content was determined by the FolinCiocalteau method [11]. Extracts $(0.5 \mathrm{ml}, 1.6 \mathrm{mg} \mathrm{ml}-1)$ were mixed with $2.5 \mathrm{ml}$ of $0.2 \mathrm{~N}$ Folin-Ciocalteau reagent for $5 \mathrm{~min}$ and $2.0 \mathrm{ml}$ of $75 \mathrm{~g} \mathrm{l}-1$ sodium carbonate was then added. The absorbance of the reaction was measured spectrophotometrically at $760 \mathrm{~nm}$ after $2 \mathrm{~h}$ of incubation at room temperature (r.t.). Results were expressed as gallic acid equivalents.

\section{DPPH Radical-Scavenging Activity}

The stable DPPH was used for the determination of free radical scavenging activity of the extract [12]. Different concentrations of extracts were added at an equal volume, to the methanolic solution of DPPH $(100 \mu \mathrm{M})$. After $15 \mathrm{~min}$ at room temperature, the absorbance was recorded at $517 \mathrm{~nm}$. The experiment was repeated three times. Vitamin $\mathrm{C}, \mathrm{BHA}$ and quercetin were used as 
standard controls. IC ${ }^{50}$ values denote the concentration of sample, which is required to scavenge $50 \%$ of DPPH free radicals.

\section{Result and Discussion \\ DPPH Radical-Scavenging Activity}

Phenols and polyphenolic compounds, such as flavonoids, are widely found in food products derived from plant sources. There are different amounts of phenols and polyphenolic compounds in these two phytoplanktons. In these two algae, there were high amounts of phenols and polyphenolic compounds. Increasing the levels of flavonoids in the daily diet may decrease the impact or occurrence of certain human diseases because they interact with various biological systems and show anti-inflammatory, hypolipidemic, hypoglycemic and antioxidant.

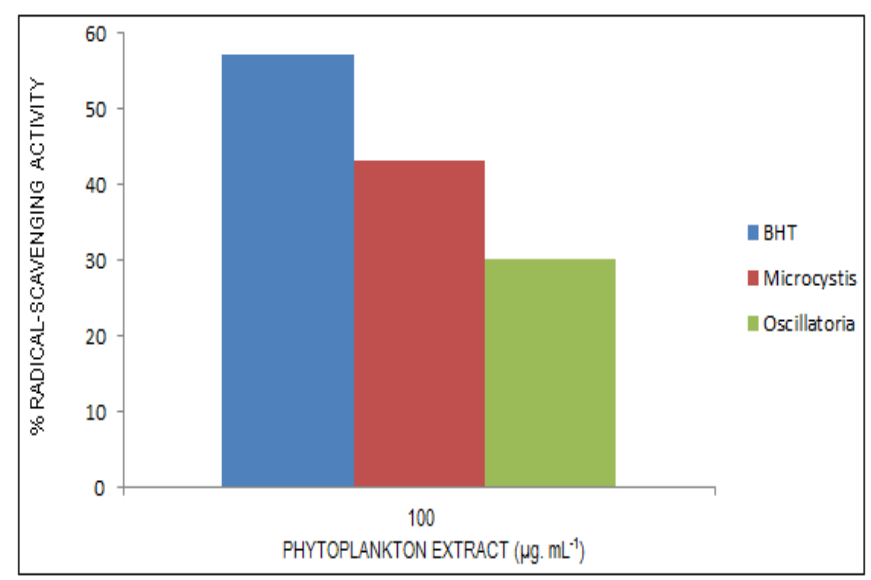

Fig. 1-

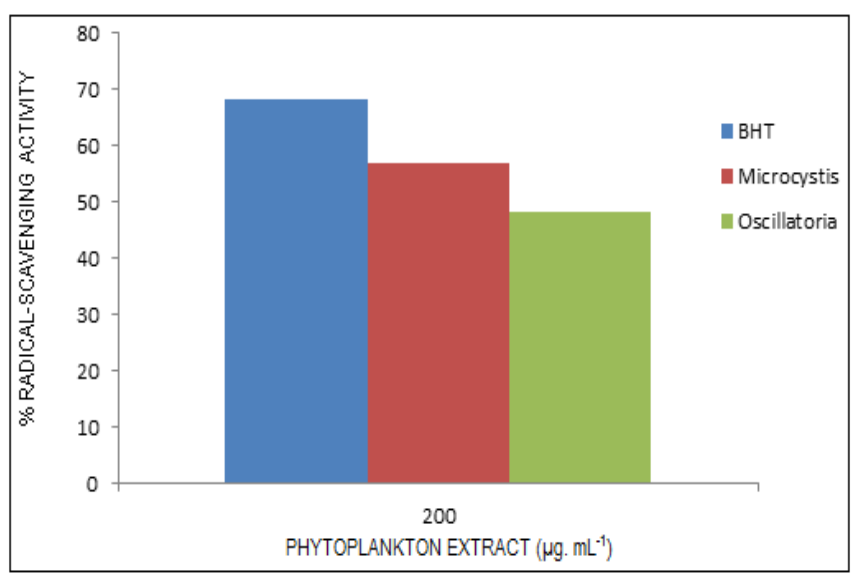

Fig. 2-

DPPH is a free radical that accepts an electron or changes from violet to yellow upon reduction by either the process of hydrogenor electron-donation. Substances which are able to perform this reaction can be considered as antioxidants and therefore radical scavengers [13]. IC ${ }^{50}$ for the DPPH radicalscavenging activity was $920 \pm 42 \mu \mathrm{g} \mathrm{ml}-1$. The IC ${ }^{50}$. Phenol and flavonoid contents of these two algae may have led to its good DPPH-scavenging activity. The correlation between total phenol contents and antioxidant activity has been widely studied in different foodstuffs such as fruit and vegetables. Radical-scavenging activity assayed by the DPPH method. Samples from all of the studied species exhibited radical-scavenging activity. Among them, the extracts of $M$. aeruginosa $(43.26 \%)$ exhibited higher activity than those of and Oscillatoria sp. (30.17\%) (Fig. 1) in $100 \mu \mathrm{g}$ of extracts,but in 200 (Fig. 2) and $300 \mu \mathrm{g}$ (Fig. 3) of extracts of Microcystis sp. exhibited $57.91 \%$ and $78.62 \%$ respectively. Similarly in $200 \mu \mathrm{g}$ and $300 \mu \mathrm{g}$ Oscillatoria sp. exhibited $48.87 \%$ and $58.02 \%$ respectively.

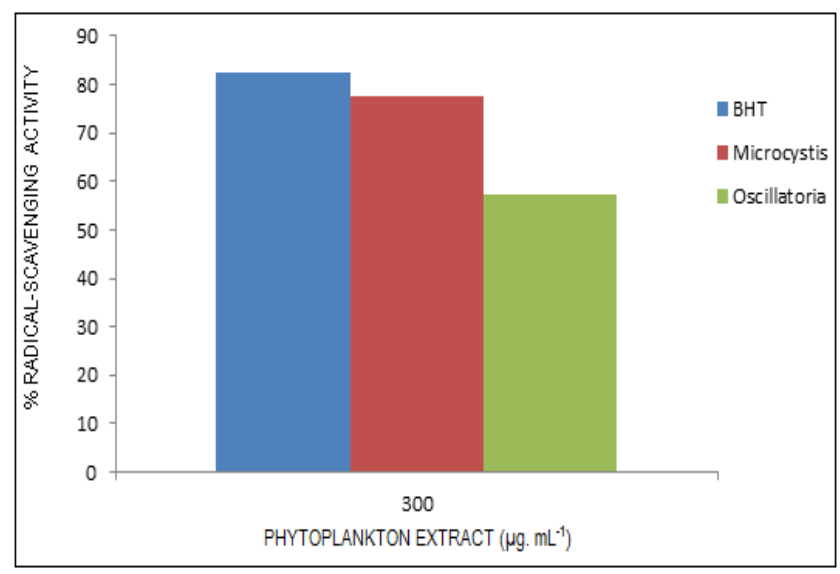

Fig. 3-

\section{Conclusions}

In conclusion, the methanolic and aqueous extracts of the examined Microcystis sp and. Oscillatoria sp.contain antioxidative compounds that can strongly scavenge ROS such as superoxide anion, hydroxyl radical, hydrogen peroxide and DPPH free radical. These results indicate that the methanolic and aqueous extracts of these two species can be used as potential natural antioxidants in foods and pharmaceutical industries. However, the responsible compounds related to the antioxidant activity of extract of these two species are not yet cleared. Therefore, further studies are required in order to identify the antioxidant compound of these two microalgae extracts. These two species showed good but different levels of antioxidant activities in some models studied. The extracts had high amount of phenols and polyphenolic compounds. Phenols and polyphenolic compounds were in very good amount and higher than those in some plants [14]. DPPH-scavenging activity showed potent activity. Identification of the antioxidant compounds of these two extracts will lead to their evaluation in considerable commercial potential in medicine, food production and in the cosmetic industry.

\section{Acknowledgement}

The authors express their deep sense of gratitude to Head, P.G. Department of Botany, Berhampur University for providing necessary laboratory facilities.

\section{References}

[1] Yagi K. (1987) Chem. Phys. Lipids, 45, 337-341.

[2] Zainol M.K., Abd-Hamid A., Yusof S. and Muse R. (2003) J. Agric. Food Chem., 48, 2008-2016.

[3] Robinson E.E., Maxwell S.R.J. and Thorpe G.H.G. (1997) Free Radical Res., 26, 291-302

[4] Karawita R., Siriwardhana N., Lee K.W., Heo M.S., Yeo I.K., Lee Y.D. and Jeon Y.J. (2005) Eur. Food Res. Technol., 220, 363-371. 
[5] Alho H. and Leinonen J. (1999) Enzymol., 299, 3-15.

[6] Lemberkovices É., Czinner E., Szentmihályi K., Balázs A. and Szöke É. (2002) Food Chem., 78, 119-127.

[7] Park P.J., Heo S.J., Park E.J., Kim S.K., Byun, H.G., Jeon B. T. and Jeon Y.J. (2005) J. Agric. Food. Chem., 53, 6666-72.

[8] Jun W.J., Han B.K., Yu K.W., Kim M.S., Chang I.S., Kim H.Y. and Cho H.Y. (2001) Food Chem., 75, 439-444.

[9] Lu Y. and Foo Y.L. (2000) Food Chem., 68, 81-85.

[10]Bligh E.G. and Dyer W.J. (1959) Can. J. Biochem. Physiol., 37, 911-7.

[11]McDonald S., Prenzler P.D., Autolovich M., Robards K. (2001) Food Chem., 73, 73-84.

[12]Koleva II, Van Beek T.A., Linssen J.P.H. (2002) Phytochemical Analysis, 13, 8-17.

[13]Ebrahimzadeh M.A., Nabavi S.F., Nabavi S.M., Eslami B. (2010a) Grasas Aceites., 61,30-36.

[14]Ebrahimzadeh M.A., Nabavi S.F., Nabavi S.M., Eslami B., Dehpour A.A. (2010b) Afr. J. Biotechnol., 9(34), 5548-5555. 